\title{
Ergebnis zur Weiterentwicklung der EE-Wärme-Quote
}

Wir befinden uns in einer Phase des Übergangs hin zu einer klimaneutralen Wirtschaftsund Gesellschaftsordnung. Anders als bei vielen historischen Transformationsprozessen bleibt uns nur noch wenig Zeit, handelt es sich zudem um eine weltweite Aufgabe und haben wir andererseits alle Möglichkeiten in der Hand, einen friedlichen Weg zu beschreiten. Führt man sich diese Tatsachen vor Augen, scheinen die gesetzesübergreifende Harmonisierung der EE-Begriffe, die Erweiterung des Anwendungsbereichs der EE-Wärme-Quote und eine moderate dem Stand der Technik folgende schrittweise Erhöhung der Quote nicht die härtesten denkbaren regulatorischen Eingriffe zu sein. Natürlich sollte die Politik nicht unter den Vorzeichen eines dauerhaften Krisenzustandes - hier der Klimakrise - Entscheidungen treffen. Aber auch ohne Menetekel sind die vorgeschlagenen Weiterentwicklungen rechtspolitisch sinnvoll, bilden sie doch die Vielfalt der Wärmeversorgung des Gebäudesektors über Ersatzmaßnahmen und Kombinationsmöglichkeiten ab, ohne das Gesamtziel der Steigerung des EE-Anteils und der Verringerung der Treibhausgasemissionen aus dem Blick zu verlieren.

(C) Der/die Autor(en), exklusiv lizenziert durch Springer Fachmedien Wiesbaden GmbH, ein Teil von Springer Nature 2021

S. Schäfer-Stradowsky, Das Recht der erneuerbaren Energien zur Wärmeversorgung des Gebäudesektors, Schriftenreihe des Instituts für Klimaschutz, Energie und Mobilität, https://doi.org/10.1007/978-3-658-35016-1_16 